\title{
Apoptotic proteins as prognostic markers and indicators of radiochemosensitivity in stage II/III rectal cancers
}

\author{
C. Fucini*, L. Messerini†, C. Saievał, L. Orzalesi*, V. Carroni* and N. Bartolini* \\ *Department of Medical and Surgical Critical Care, Section of General and Oncological Surgery, †Department of Human Pathology and Oncology, \\ University of Florence and $\ddagger$ Molecular and Nutritional Epidemiology Unit, ISPO, Florence, Italy \\ Received I5 December 2010; accepted 12 May 201 I; Accepted Article online 16 August 20II
}

\begin{abstract}
Aim The expression of pro-apoptotic (Bax) and antiapoptotic (mutated p53, Bcl-2, Bclxl) proteins was determined retrospectively using immunohistochemistry in pre-treatment biopsy samples from patients with rectal cancer treated with or without preoperative chemoradiation to investigate their role as prognostic markers and indicators of radiochemosensitivity.

Method Biopsy samples from 67 patients operated for stage II/III rectal cancer and enrolled in an active followup programme were examined 8-10 years after surgery. Thirty-three had been treated with immediate surgery followed, in selected cases, by adjuvant postoperative chemoradiation. Thirty-four had preoperative chemoradiation. Immunohistochemical staining was carried out using an automated immunostainer on sections of paraffin-embedded tissue.
\end{abstract}

Results Independent prognostic factors for rectal cancer death were pN status (hazard ratio 3.82; 95\% CI $1.67-$ 8.73 ) and a high level of Bclxl positivity (hazard ratio
4.75 ; 95\% CI 2.10-10.72) according to multivariate regression analysis by stepwise selection. Bax expression was associated with downstaging and higher survival in irradiated patients $(P=0.0004)$.

Conclusion Pretreatment evaluation of apoptotic Bax and anti-apoptotic Bclxl factors in biopsy samples of stage II/III rectal cancers may be helpful in selecting tumours that will respond to chemoradiation or in identifying patients who will have limited benefit from chemoradiation and should therefore be selected for a more aggressive systemic regimen.

Keywords Rectal cancer, apoptosis, chemoradiation

\section{What is new in this paper}

The expression of pro-apoptotic and anti-apoptotic proteins was examined in pretreatment biopsy samples of patients with rectal cancer treated with or without preoperative chemoradiation to investigate their possible role as prognostic indicators of radiochemosensitivity.

\section{Introduction}

Preoperative chemoradiation is increasingly used in the treatment of operable but advanced (clinical T3) rectal cancer. The aim is to reduce local recurrence, improve survival and allow a larger number of sphincter-saving procedures. Despite the occurrence of adverse effects, including grade II/III toxicity (NCI scale), satisfactory

Correspondence to: Bartolini Niccolò, MD, Department of Medical and Surgical Critical Care, Section of General and Oncological Surgery, University of Florence, Viale Morgagni no. 85, 5014I Florence, Italy.

E-mail: n.bartolini@gmail.com

This study is an original article and has been presented in part at the IIOth National Congress of the Italian Society of Surgery, October 2008. No publication contains material that appears in this article. results have been reported in single-centre experiences and clinical trials $[1,2]$. The tumour regression and socalled 'downstaging' obtained by neoadjuvant treatment in $50-60 \%$ of cases appear to be related to a better oncological outcome and increased number of sphinctersaving operations [3-6].

However, there is a limited or no response to chemoradiation in a significant number of patients $(40-50 \%)$, and the prognosis seems to be particularly poor [5-8]. Cancer patients with persisting positive lymph nodes after chemoradiation (more than $50 \%$ of $\mathrm{N}+$ tumours identified by preoperative endorectal ultrasound) have an unfavourable outcome [6,7]. Thus a number of patients with stage II/III low rectal cancer will not benefit from preoperative chemoradiation, with 25$30 \%$ of them experiencing toxicity. It would be useful to 
have biological markers of prognostic significance and of sensitivity to chemoradiation to avoid unnecessary and potentially harmful complications in these patients [9].

Many biomolecular prognostic markers and markers of sensitivity to neoadjuvant treatment in rectal cancer have been identified and some, like endothelial growth factor receptor microsatellite instability, wild K-ras, are increasingly used in clinical practice [10-13].

Several studies have aimed to evaluate the usefulness of markers of apoptosis, the mechanism controlling the development and progression of a cancer, in relation to the disruption of cell cycle control and programmed cell death [10]. The disruption of this regulatory mechanism, particularly regarding cell death signalling, has a major impact on the resistance of malignant tumours to radiotherapy and chemotherapy [14].

The apoptotic process is regulated by various proteins at different levels. Proteins of the Bcl-2 family are central regulators of apoptosis initiated at the mitochondrial level $[15,16]$. The Bcl-2 family includes anti-apoptotic (Bcl-2 and Bclxl) and pro-apoptotic proteins (Bax). The balance and interactions between pro-apoptotic and antiapoptotic proteins regulates the apoptotic machinery [17].

Another protein involved in the apoptotic process is the p53 tumour suppressor gene. This molecule plays a central role in promoting genomic stability and tissue homeostasis [18]. Wild-type p53 induces apoptosis in damaged cells, although the marker usually detected with immunohistochemistry in clinical studies is the mutated form which has lost this activity. Mutated p53 suppresses apoptosis and confers chemotherapy and radiotherapy resistance to cancer cells [18].

The present study examined the expression of one pro-apoptotic (Bax) and three anti-apoptotic (p53, Bcl-2, $\mathrm{Bclxl})$ proteins using immunohistochemistry in pretreatment biopsy samples from preoperatively non-irradiated and irradiated rectal carcinoma patients. Patients were operated on in a period in which neoadjuvant chemoradiation for T3 cancer was not routinely adopted in our department. The aim was to investigate the role of the proteins as potential indicators of resistance or sensitivity to preoperative chemoradiation.

\section{Method}

The study was approved by the ethical committee of the Azienda Ospedaliera Universitaria Careggi, Florence. Pretreatment biopsy samples from the tumour from 67 consecutive patients (44 men) with adenocarcinoma of the rectum, treated over a 3 -year period, were examined by immunohistochemical staining 8-10 years after surgery.
All patients had had a tumour located at less than $12 \mathrm{~cm}$ from the anal verge, classified at presentation as T3 by clinical examination, endorectal sonography, abdominopelvic CT scan and, at times, MRI with endorectal coil. Lymph nodal evaluation was not performed at that time.

Preoperative chemoradiation was offered to all patients after explaining the goals and risks of neoadjuvant $v s$ a more selective adjuvant treatment. Thirty-three patients (group I) preferred to undergo immediate surgery followed by adjuvant chemoradiation when indicated, while 34 (group II) accepted preoperative neoadjuvant chemoradiotherapy. Eventually all patients were operated on undergoing a curative total mesorectal excision. Patients in group II were operated on 6-9 weeks after the completion of treatment. Patients in group I with positive lymph nodes received adjuvant chemoradiation.

Preoperative chemoradiation therapy fields included the primary tumour, the adjacent lymph node drainage area in the pelvis and the regional nodes along the superior haemorrhoidal vessels. The tumour dose was 45 Gy delivered in 25 daily fractions of 1.8 Gy. Chemotherapy consisted of fluorouracil $\left(5-\mathrm{FU}, 375 \mathrm{mg} / \mathrm{m}^{2}\right.$ bolus daily for 5 days) and leucovorin ( $\mathrm{LV}, 10 \mathrm{mg} / \mathrm{m}^{2}$ daily for 5 days). These were administered concurrently during the first 5 days and on days 29-33 of radiation therapy.

Postoperative adjuvant chemoradiation was started 4-6 weeks after surgery with chemotherapy given for two cycles every 28 days in the same dosage as above. After two cycles of 5-FU plus LV, radiotherapy was initiated in daily fractions of 2 Gy over 5 weeks to a total dose of 54 Gy. All patients with positive nodes in groups I and II were planned to receive four extra cycles of postoperative chemotherapy. Follow-up included clinical examination, CEA monitoring, colonoscopy and CT of abdomen and thorax. One patient in group II died in the perioperative period and was excluded from the study.

\section{Immunohistochemical assay}

Staining was conducted using an automated immunostainer (Ventana NexES; Ventana MedicalSystems, Tucson, Arizona, USA) on $4-\mu \mathrm{m}$ thick sections of paraffin-embedded tissue. Sections were deparaffinized in xylene and rehydrated in a descending ethanol series. Endogenous peroxidase activity was blocked by immersion for $10 \mathrm{~min}$ in phosphate-buffered saline ( $\mathrm{pH} 7.4)$. The primary antibodies used in the study were as follows: (1) anti-p53 monoclonal antibody (dilution 1:100; clone DO-7, Dako SA, Glostrup, Denmark); (2) anti-Bcl-2 monoclonal antibody (dilution 1:50; clone 124, Dako) recognizing mutant protein; (3) anti-Bclxl monoclonal 
antibody [dilution 1:50 (Zymed, San Francisco, California, USA) in $0.3 \%$ hydrogen peroxide in methanol solution, followed by a single wash in phosphate]; and (4) anti-Bax polyclonal antibody (dilution 1:50; code A3533, Dako). The immunostaining was developed using $3,3^{\prime}$-diaminobenzidine as chromogen. Appropriate positive and negative controls were added to each automated immunohistochemistry run to confirm antibody specificity.

After immunostaining, in each case the entire section was examined by light microscopy at a magnification $100 \times$ by one pathologist (LM) without knowledge of the patient's outcome. The percentage of immunostained cells was originally recorded as follows: 0 (none or $<10 \%) ; 1+(10-25 \%) ; 2+(26-50 \%) ; 3+(51-75 \%) ;$ $4+(76-100 \%)$. To simplify statistical analysis, however, these were reduced to three groups: negative (none or $<10 \%$ ), medium positive $(10-50 \%)$ or high positive $(>50 \%)$. The different levels of Bclxl are shown in Fig. 1 and the high levels of Bcl-2, Bax and p53 in Fig. 2.

\section{Statistical analysis}

For regression analyses, we used for each marker a medium positivity level and a high positivity level which were compared with the negative level. Selected characteristics were compared in the groups using Fisher's exact test, the chi squared or the Mann-Whitney test as appropriate. Survival was calculated from the date of the first treatment to the date of the last follow-up or death. Local disease-free survival was calculated from the date of first treatment to the date of recurrence. Crude survival was estimated using the Kaplan-Meier method and differences between patient groups were assessed by the log-rank test. Comparisons in survival were made using the Cox proportional hazards regression model. Estimated relative risks of dying were expressed as adjusted hazard ratios (HRs) with 95\% CI. A regression model with stepwise selection was performed to identify the major significant predictors of death. Statistical results were considered significant at a $P$-value of $<0.05$. All statistical tests were performed using SAS software (Florence, Italy).

\section{Results}

Preoperative assessment correctly staged T3 cancers in 31 (94\%) patients in group I $(n=33)$. One patient had a T2 and another a T4 cancer. In group II $(n=33)$ the results of pathological examination of the resected specimen showed eight T0 (24\%), one TI (3\%), eight T2 (24\%), 16 T3 $(49 \%)$ tumours. The individual characteristics of the 66 patients, nodal status and results of treatments are shown in Table 1. Twenty-two patients in group I had
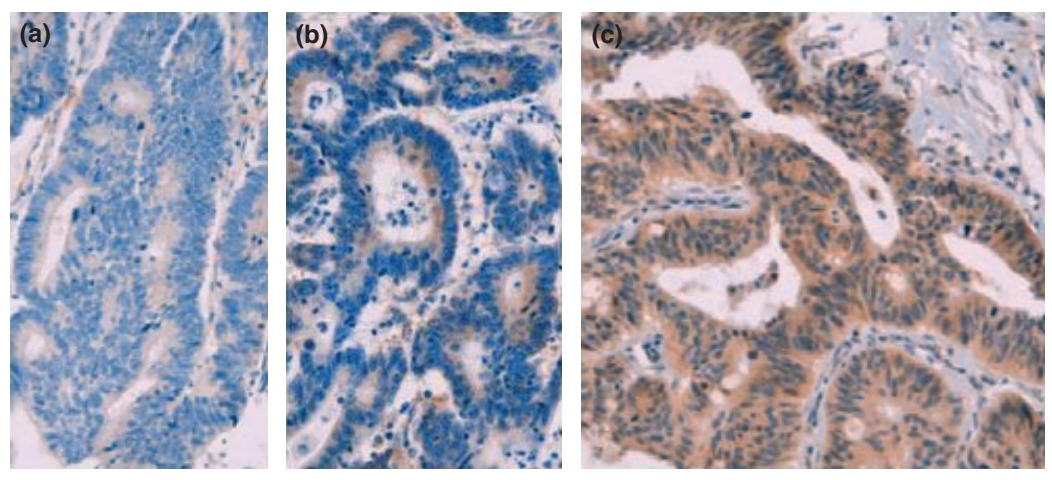

Figure I Examples of three levels of Bclxl immunostaining: (a) negative (none or $<10 \%$ ); (b) medium positive (10-50\%); (c) high positive $(>50 \%)$.
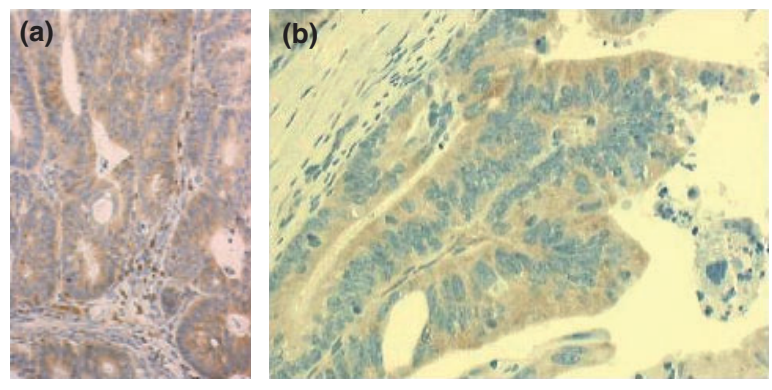

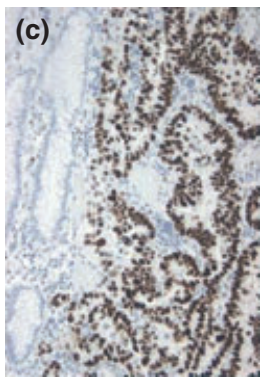

Figure 2 Examples of high positivity immunostaining: (a) Bcl-2; (b) Bax; (c) p53. 
Table I Distribution of 66 rectal cancer patients according to individual characteristics and results of treatment.

\begin{tabular}{|c|c|c|c|}
\hline Characteristics & $\begin{array}{l}\text { Group I, } \\
N(\%)\end{array}$ & $\begin{array}{l}\text { Group II, } \\
N(\%)\end{array}$ & $P$-valuex \\
\hline \multicolumn{4}{|l|}{ Sex } \\
\hline Male & $22(66.7)$ & $21(63.6)$ & \multirow[t]{2}{*}{1.0} \\
\hline Female & $11(33.3)$ & $12(31.8)$ & \\
\hline \multicolumn{4}{|l|}{ Age, years } \\
\hline$\leq 55$ & - & $8(24.2)$ & \multirow[t]{4}{*}{0.01} \\
\hline $56-65$ & $9(27.3)$ & $11(33.3)$ & \\
\hline $66-75$ & $14(42.4)$ & $9(27.3)$ & \\
\hline$>75$ & $10(30.3)$ & $5(15.2)$ & \\
\hline Mean age $( \pm \mathrm{SD})$ & $70.5( \pm 7.2)$ & $63.6( \pm 9.4)$ & 0.004 \\
\hline \multicolumn{4}{|l|}{$\mathrm{pN}$} \\
\hline N0 & $22(66.7)$ & $23(69.7)$ & \multirow[t]{3}{*}{0.58} \\
\hline $\mathrm{Nl}$ & $8(24.2)$ & $9(27.3)$ & \\
\hline N2 & $3(9.1)$ & $1(3.0)$ & \\
\hline \multicolumn{4}{|l|}{ Surgery } \\
\hline $\mathrm{AP}$ & $5(15.2)$ & $8(24.2)$ & \multirow[t]{3}{*}{0.04} \\
\hline $\mathrm{AR}$ & $8(54.5)$ & $8(24.2)$ & \\
\hline CA & $10(30.3)$ & $17(51.6)$ & \\
\hline \multicolumn{4}{|c|}{ Distance from anal verge $(\mathrm{cm})$} \\
\hline$\geq 6$ & $13(39.4)$ & $7(21.2)$ & \multirow[t]{2}{*}{0.18} \\
\hline$<6$ & $20(60.6)$ & $26(78.8)$ & \\
\hline \multicolumn{4}{|l|}{ Local recurrence } \\
\hline Negative & $29(87.9)$ & $31(93.9)$ & \multirow[t]{2}{*}{0.69} \\
\hline Positive & $4(12.1)$ & $2(6.1)$ & \\
\hline \multicolumn{4}{|l|}{ Distant metastasis } \\
\hline Negative & $26(78.7)$ & $20(60.6)$ & \multirow[t]{2}{*}{0.09} \\
\hline Positive & $7(21.3)$ & $13(39.4)$ & \\
\hline \multicolumn{4}{|l|}{ Status } \\
\hline Alive & $23(69.7)$ & $16(48.5)$ & \multirow[t]{3}{*}{0.13} \\
\hline Dead & $10(30.3)$ & $17(51.5)$ & \\
\hline Total & $33(50.0)$ & $33(50.0)$ & \\
\hline
\end{tabular}

$\mathrm{AP}$, abdominoperineal excision; $\mathrm{AR}$, anterior resection; $\mathrm{CA}$, coloanal anastomosis.

* $P$-value from Fisher exact test, chi squared for trend or MannWhitney test, as appropriate.

surgery alone, 11 had postoperative chemoradiation because of lymph node involvement $(\mathrm{N}+)$, in two of them there were infiltration of circular resection margin.

Of the 66 patients, $43(65 \%)$ were men. The mean age was $67(S D \pm 9)$ years. Patients in group I were younger $(P=0.004)$. There were no differences between the groups in the distance of the tumour from the anal verge or in the proportion of sphincter-sparing procedures ( $86 \%$ vs $75 \%)$. No patient was lost to follow-up. At the last follow-up (mean $105.5 \pm 39.6$ months), 27 (40.9\%) patients had died. The incidence of local recurrence $(2 \%$ vs $4 \% ; P=0.69)$, distant metastases $(13 \%$ vs $7 \%$; $P=0.09)$ and survival $(P=0.13)$ was not statistically different between the groups. The pretreatment distribution of markers was also similar, apart from the absence
Table 2 Distribution of 66 patients with rectal cancer according to marker expression in groups I and II.

\begin{tabular}{lrrr}
\hline Marker & Group I, & \multicolumn{1}{c}{ Group II, } & \\
& $N(\%)$ & $N(\%)$ & $P$-value* \\
p53 & & & \\
Negative & $9(27.3)$ & $7(21.2)$ & 0.61 \\
Medium positive & $13(39.4)$ & $17(51.5)$ & \\
High positive & $11(33.3)$ & $9(27.3)$ & \\
Bax & & & \\
Negative & $17(51.5)$ & $9(27.3)$ & 0.10 \\
Medium positive & $12(36.4)$ & $13(39.4)$ & \\
High positive & $4(12.1)$ & $11(33.3)$ & \\
Bcl-2 & & & \\
Negative & $8(24.2)$ & $18(54.6)$ & 0.03 \\
Medium positive & $13(39.4)$ & $15(45.4)$ & \\
High positive & $12(36.4)$ & 0 & \\
Bclxl & & & \\
Negative & $10(30.3)$ & $17(51.5)$ & 0.21 \\
Medium positive & $14(42.4)$ & $9(27.3)$ & \\
High positive & $9(27.3)$ & $7(21.2)$ & \\
Total & $33(50.0)$ & $33(50.0)$ & \\
\hline
\end{tabular}

Expression was classified as negative (none or $<10 \%$ of tumour cells stained), medium positive ( $10-50 \%$ of tumour cells stained) or high positive (> $50 \%$ of tumour cells stained).

${ }^{*} P$-value from chi squared.

of patients with a high level of positivity for $\mathrm{Bcl}-2$ in the chemoradiation group (Table 2).

In group I, despite the disparity in mortality between $11 \mathrm{~N}+$ and $22 \mathrm{~N}$ - patients (45.5\% vs 22.7\%) the difference was not significant (Table 3 ). However, a statistically significantly lower survival was observed in patients with high levels of positivity for p53 $(P=0.003), \mathrm{Bcl}-2$ $(P=0.01)$ and Bclxl $(P=0.003)$. A borderline association between Bax expression and improved survival was also seen $(P=0.06)$.

Multivariate regression analysis by stepwise selection showed a high level of positivity of Bclxl (HR 3.99; 95\% CI 1.03-1542) and p53 (HR 4.99; 95\% CI 1.16-21.44) to be significant independent prognostic factors for lower survival (Table 3 ).

In node-negative patients of group I $(n=22)$ no significant relationship between the level of the markers and survival was observed. On the contrary, in nodepositive patients of the same group $(n=11)$ a high expression of Bclxl was associated with a significantly higher risk of death $(P=0.01)$. There was an almost statistically significant $(P=0.075)$ relationship between Bax level and node-positive patients with lower mortality.

In group II, pN status, p53 and Bclxl high level expression emerged as significant predictors of death $(P=0.0001, \quad P=0.03$ and $P=0.004)$, while $B$ ax 
Table 3 Clinico-pathological characteristics and marker expression in relation to specific survival in the two groups of patients.

\begin{tabular}{|c|c|c|c|c|c|}
\hline & \multirow[b]{2}{*}{ At start } & \multicolumn{2}{|l|}{ Follow-up } & \multirow[b]{2}{*}{ Log-rank test* } & \multirow[b]{2}{*}{$\operatorname{HR}(95 \% \mathrm{CI}) \dagger$} \\
\hline & & No. of deaths & Specific survival & & \\
\hline \multicolumn{6}{|l|}{ Group I } \\
\hline \multicolumn{6}{|l|}{$\mathrm{pN}$} \\
\hline NO & 22 & 5 & $75.8(51.1-89.2)$ & 0.13 & - \\
\hline $\mathrm{Nl}$ & 8 & 3 & $58.3(18.0-84.4)$ & & \\
\hline $\mathrm{N} 2$ & 3 & 2 & $33.3(0.9-77.4)$ & & \\
\hline \multicolumn{6}{|l|}{ p53 } \\
\hline Negative & 9 & 2 & $77.8(36.5-93.9)$ & 0.003 & $1 \ddagger$ \\
\hline Medium positive & 13 & 1 & $92.3(56.6-98.9)$ & & $0.37(0.03-4.11)$ \\
\hline High positive & 11 & 7 & $25.3(4.1-55.2)$ & & $4.94(1.01-24.34)$ \\
\hline \multicolumn{6}{|l|}{ Bax } \\
\hline Negative & 17 & 8 & $47.1(20.9-69.5)$ & 0.06 & - \\
\hline Medium positive & 12 & 2 & $82.5(46.1-95.3)$ & & \\
\hline High positive & 4 & 0 & 100 & & \\
\hline \multicolumn{6}{|l|}{$\mathrm{Bcl}-2$} \\
\hline Negative & 8 & 0 & 100 & 0.01 & - \\
\hline Medium positive & 13 & 3 & $76.9(44.2-91.9)$ & & $1 \dagger$ \\
\hline High positive & 12 & 7 & $33.0(8.4-60.9)$ & & $3.44(0.88-13.44)$ \\
\hline \multicolumn{6}{|l|}{ Bclxl } \\
\hline Negative & 10 & 1 & $87.5(38.7-98.1)$ & 0.003 & $1 \ddagger$ \\
\hline Medium positive & 14 & 3 & $77.9(45.9-92.3)$ & & $2.26(0.24-21.73)$ \\
\hline High positive & 9 & 6 & $25.4(3.8-56.4)$ & & $11.64(1.39-97.31)$ \\
\hline Total & 33 & 10 & $67.3(47.6-81.0)$ & & \\
\hline \multicolumn{6}{|l|}{ Group II } \\
\hline \multicolumn{6}{|l|}{$\mathrm{pN}$} \\
\hline NO & 23 & 9 & $59.8(37.37-82.4)$ & 0.0001 & $1 \ddagger$ \\
\hline $\mathrm{Nl}$ & 9 & 7 & $14.8(0-40.9)$ & & $6.49(2.17-19.41)$ \\
\hline N2 & 1 & 1 & $0(-)$ & & $23.70(2.19-256.7)$ \\
\hline \multicolumn{6}{|l|}{ p53 } \\
\hline Negative & 7 & 2 & $68.6(32.1-100)$ & 0.03 & $1 \ddagger$ \\
\hline Medium positive & 17 & 7 & $54.4(28.9-79.9)$ & & $1.40(0.29-6.77)$ \\
\hline High positive & 9 & 8 & $16.7(0-44.4)$ & & $4.27(0.90-20.41)$ \\
\hline \multicolumn{6}{|l|}{ Bax } \\
\hline Negative & 9 & 7 & $16.7(10-44.4)$ & 0.0004 & $1 \ddagger$ \\
\hline Medium positive & 13 & 6 & $69.6(33.3-87.7)$ & & $0.16(0.05-0.53)$ \\
\hline High positive & 11 & 4 & $46.2(4.3-88.0)$ & & $0.13(0.03-0.49)$ \\
\hline \multicolumn{6}{|l|}{$\mathrm{Bcl}-2$} \\
\hline Negative & 18 & 7 & $51.2(22.0-80.4)$ & 0.11 & $1 \ddagger$ \\
\hline Medium positive & 15 & 10 & $37.5(12.1-62.9)$ & & $2.16(0.82-5.70)$ \\
\hline High positive & - & - & - & & \\
\hline \multicolumn{6}{|l|}{ Bclxl } \\
\hline Negative & 17 & 6 & $54.1(23.84 .3)$ & 0.004 & $1 \ddagger$ \\
\hline Medium positive & 9 & 5 & $53.3(19.4-87.3)$ & & $1.93(0.58-6.40)$ \\
\hline High positive & 7 & 6 & $14.3(0-40.8)$ & & $5.96(1.87-19.02)$ \\
\hline Total & 33 & 17 & $23.9(2.0-57.9)$ & & \\
\hline
\end{tabular}

*From univariate survival analysis by the Kaplan-Meier method. †From Cox regression analysis.

$\ddagger$ Reference category.

expression was associated with higher survival $(P=0.0004)$. Multivariate regression analysis by stepwise selection indicated that $\mathrm{pN}$ status (HR 5.95; 95\% CI
1.92-18.46) and a high level of Bclxl positivity (HR 3.51; 95\% CI 1.16-10.59) were significant independent prognostic factors of death from rectal cancer. 
When all 66 patients were submitted to multivariate regression analysis by stepwise selection, including the items 'preoperative chemoradiation' and 'postoperative chemoradiation', only pN status (HR 3.82; 95\% CI 1.678.73 ) and a high level of Bclxl positivity (HR 4.75; 95\% CI 2.10-10.72) were found to be independent prognostic factors for death from rectal cancer. The survival rate according to Bclxl expression is shown in Fig. 3 .

Analysis of $\mathrm{pN}$ status with each marker showed a significant interaction with a high level of Bclxl positivity in the whole series (HR I7.10; 95\% CI 6.17-47.54), and also in group analysis (group I: HR 16.3; 95\% CI 3.5474.86; group II: HR 21.59; 95\% CI 2.87-162.57).

\section{Downstaging}

Seventeen patients in group I showed a downstaging effect (from clinical T3 to pathological T2, T1, T0). All downstaged cases were Bax positive and 15/17 (88.2\%) were p53 positive. Bax positivity was significantly associated with downstaging $(P=0.001)$ and longer survival. A trend toward better survival was also demonstrated by downstaged patients with a negative or very low expression of p53 (50\% vs $28 \%$ ). Five-year survival was significantly better in downstaged patients (log-rank test $P=0.016)$ although in the long term this difference seemed to disappear (log-rank test $P=0.32$ ) (Fig. 4).

\section{Local recurrence}

Only six local recurrences were found in the whole series (four in group I, two in group II), occurring at 23 (6-72) months. There was no relationship between the incidence of local recurrence and the level of any of the markers.

\section{Discussion}

Despite possible bias due to the small number of patients in the study, the high level of significance of some of the results is likely to indicate real associations between the markers of apoptosis and response to chemoradiotherapy and survival.
Figure 3 Kaplan-Meier survival curve for 66 rectal cancer patients according to Bclxl expression.

Figure 4 Kaplan-Meier survival curve for 33 patients in group II according to the presence or absence of downstaging.
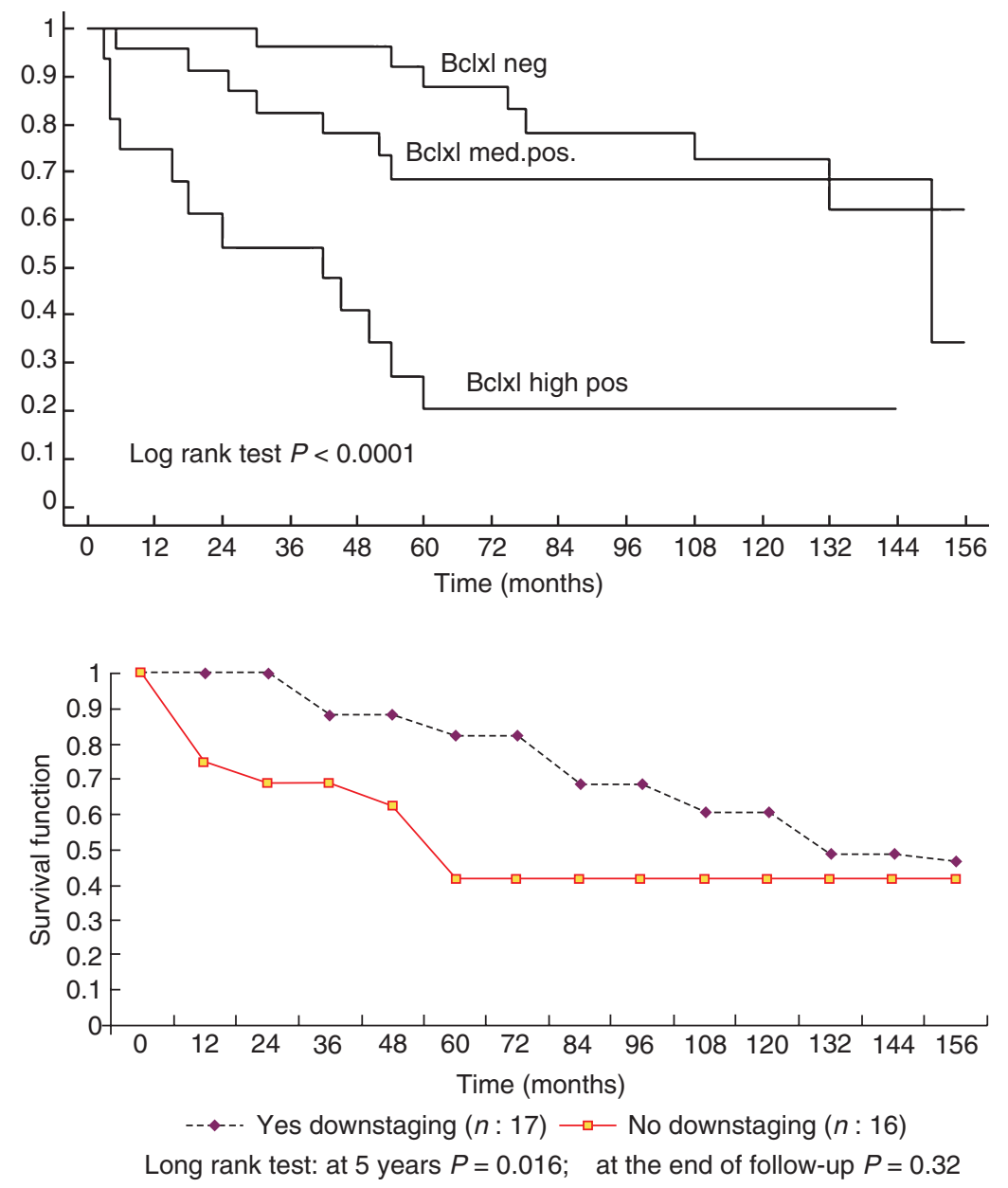
p53 has been the most studied prognostic marker and predictor of response to chemoradiation in rectal cancer [18-20]. Various techniques have been used to detect p53, including immunohistochemistry, single-strand conformational polymorphism analysis and direct p53 gene sequencing. Immunochemistry has been the most frequently used method [21]. Regardless of the evaluation method, the correlation between p53 and response to radiotherapy, rate of local recurrence and disease-free overall survival observed by some workers [21] has not been confirmed by others $[22,23]$.

In the present study using univariate analysis, overexpression of the marker appeared to be significantly related to worse survival in both groups of patients when the rate of stained cells exceeded $50 \%$. However, p53 was no longer an independent prognostic factor according to multivariate regression analysis of all patients. In addition, no significant correlation was observed between p53 expression and response to radiochemotherapy even though $88.4 \%$ of downstaged patients were p53 positive.

Results reported in the literature regarding Bax protein are also controversial. Some authors report that Bax overexpression in stage Il cancer is a marker of poor prognosis and higher local recurrence [20], but others have found no association $[20,24]$. In more recent studies, however, Bax appeared to be a very reliable predictive marker for therapeutic response to preoperative radiochemotherapy $[25,26]$ with a higher expression in complete than in partial responders. In other reports a high expression of Bax protein and low expression of p53 were related to a better prognosis in stage III cancer [27] and, according to univariate and multivariate analysis, Bax expression was an independent prognostic indicator of longer local recurrence-free interval and disease-free survival [28].

Our results are in agreement with the more recent studies. We found that Bax was positively related with response to preoperative neoadjuvant chemoradiotherapy and overall survival (Table 3).

With regard to $\mathrm{Bcl}-2$ our results are similar to data in the literature [15]. No particular value was found to predict response to chemoradiation, although some authors have found that an elevated tissue $\mathrm{Bcl}-2 / \mathrm{Bax}$ ratio is related to increased tumour resistance to adjuvant radiotherapy [29]. One indication of prognosis was found in patients having immediate surgery who had a high rate of Bcl-2 stained cells, according to univariate analysis. The role of $\mathrm{Bcl}-2$ in patients having chemoradiotherapy is less clear since in the present study there was an absence of high expression of this marker in all patients. This marker may have some prognostic value but at a much lower strength than other markers.
Bclxl is the least understood marker. This antiapoptotic molecule, which seems to protect cancer cells from wild p53-mediated apoptosis [30], has recently been identified to be 10 times more effective than $\mathrm{Bcl}-$ 2 when expressed in a breast cancer line [31]. In our experience its role as a prognostic marker was relevant. According to stepwise-selected multivariate regression analysis, only $\mathrm{pN}$ status and a high level of Bclxl positivity emerged as independent prognostic factors for death due to rectal cancer. When we examined the results separately in groups I and II by multivariate regression analysis a high level of Bclxl remained the only significant independent prognostic factor although it did show a significant interaction with $\mathrm{pN}$ status.

In conclusion, a high expression of apoptotic Bax protein is correlated with a good response to preoperative chemoradiation and possibly with improved survival. Improved survival was also observed in $\mathrm{N}+$ patients who underwent surgery and postoperative adjuvant chemoradiation.

The anti-apoptotic paralogue Bclxl, when highly expressed, seems to have such a powerful prognostic influence on advanced stage II and III rectal cancer patients treated with or without preoperative chemoradiation that it overcomes the effect of the other apoptotic markers and possibly even of lymph node positivity. When a high expression of Bclxl is combined with lymph node positivity, the prognosis is particularly poor, regardless of the type of treatment administered. Since such patients formed $23 \%$ of our entire patient group, it is of the utmost importance to develop more reliable methods of preoperative nodal staging and to confirm the prognostic role of Bclxl in a prospective study. Patients suspected of being node positive before treatment who have a high expression of Bclxl in a biopsy specimen should be regarded as being at high risk of a poor outcome and should be administered more aggressive systemic treatment.

\section{References}

1 Kapiteijn E, Marijnen CA, Nagtegaal ID et al. Preoperative radiotherapy combined with total mesorectal excision for resectable rectal cancer. N Engl J Med 2001; 345: 638-46.

2 Sauer R, Becker H, Hohenberger W et al. Preoperative versus postoperative chemoradiotherapy for rectal cancer. $N$ Engl J Med 2004; 351: 1731-40.

3 Mandard AM, Dalibard F, Mandard JC et al. Pathologic assessment of tumor regression after preoperative chemoradiotherapy of esophageal carcinoma. Clinicopathologic correlations. Cancer 1994; 73: 2680-6.

4 Theodoropoulos G, Wise WE, Padmanabhan A et al. T-level downstaging and complete pathologic response after preop- 
erative chemoradiation for advanced rectal cancer result in decreased recurrence and improved disease-free survival. Dis Colon Rectum 2002; 45: 895-903.

5 Ruo L, Tickoo S, Klimstra DS et al. Long term prognostic significance of extent of rectal cancer response to preoperative radiation and chemotherapy. Ann Surg 2002; 236: 7581.

6 Rinkus KM, Russel GB, Levine EA. Prognostic significance of nodal disease following preoperative radiation for rectal cancer. Am Surg 2002; 68: 482-7.

7 Kim NK, Baik SH, Seong JS et al. Oncologic outcomes after neoadjuvant chemoradiation followed by curative resection with tumor-specific mesorectal excision for fixed locally advanced rectal cancer: impact of postirradiated pathologic downstaging on local recurrence and survival. Ann Surg 2006; 244: 1024-30.

8 Bujko K, Michalski W, Kepka L et al. Polish Colorectal Study Group. Association between pathologic response in metastatic lymph nodes after preoperative chemoradiotherapy and risk of distant metastases in rectal cancer: an analysis of outcomes in a randomized trial. Int J Radiat Oncol Biol Phys 2007; 67: 369-77.

9 Okonkwo A, Musunuri S, Talamonti N et al. Molecular markers and prediction of response to chemoradiation in rectal cancer. Oncol Rep 2001; 8: 497-500.

10 Smith FM, Reynolds JV, Miller N et al. Pathological and molecular predictors of the response of rectal cancer to neoadjuvant radiochemotherapy. Eur J Surg Oncol 2006; 32: $55-64$.

11 Bengala C, Bettelli S, Bertolini F et al. Epidermal growth factor receptor gene copy number, K-ras mutation and pathological response to preoperative cetuximab, 5-FU and radiation therapy in locally advanced rectal cancer. Ann Oncol 2009; 20: 469-74.

12 Levefre JH, Rondelli F, Mourra N et al. Lumboaortic and iliac lymphadenectomy for lymph node recurrence of colorectal cancer: prognostic value of the MSI phenotype. Ann Surg Oncol 2008; 15: 2433-8.

13 Marquardt F, Rödel F, Capalbo G et al. Molecular targeted treatment and radiation therapy for rectal cancer. Strablenther Onkol 2009; 185: 371-8.

14 Thompson CB. Apoptosis in the pathogenesis and treatment of disease. Science 1995; 267: 1456-62.

15 Hengartner MO. The biochemistry of apoptosis. Nature 2000; 407: 770-6.

16 Chao DT, Linette GP, Boise LH et al. Bcl-XL and Bcl-2 repress a common pathway of cell death. J Exp Med 1995; 182: $821-8$

17 Cory S, Huang DC, Adams JM. The Bcl-2 family: roles in cell survival and oncogenesis. Oncogene 2003; 22: 8590607.
18 Greenblatt MS, Bennett WP, Hollstein M et al. Mutations in the p53 tumor suppressor gene: clues to cancer etiology and molecular pathogenesis. Cancer Res 1994; 54: 4855-78.

19 Lowe SW, Schmitt EM, Smith SW et al.p53 is required for radiation-induced apoptosis in mouse thymocytes. Nature 1993; 362: 847-9.

20 Nehls O, Klump B, Holzmann K et al. Influence of p53 status on prognosis in preoperatively irradiated rectal carcinoma. Cancer 1999; 85: 2541-8.

21 Wynford-Thomas D. p53 in tumour pathology: can we trust immunocytochemistry? J Pathol 1992; 166: 329-30.

22 Lopez-Crapez E, Bibeau F, Thézenas $S$ et al. p53 status and response to radiotherapy in rectal cancer: a prospective multilevel analysis. Br J Cancer 2005; 92: 2114-21.

23 Saw RP, Morgan M, Koorey D et al. p53, deleted in colorectal cancer gene, and thymidylate synthase as predictors of histopathologic response and survival in low, locally advanced rectal cancer treated with preoperative adjuvant therapy. Dis Colon Rectum 2003; 46: 192-202.

24 Cascinu S, Graziano F, Catalano V et al. An analysis of p53, BAX and vascular endothelial growth factor expression in node-positive rectal cancer. Relationships with tumour recurrence and event-free survival of patients treated with adjuvant chemoradiation. Br J Cancer 2002; 86: 744-9.

25 Chang HJ, Jung KH, Kim DY et al. Bax, a predictive marker for therapeutic response to preoperative chemoradiotherapy in patients with rectal carcinoma. Hum Pathol 2005; 36: 364-71.

26 Nasierowska-Guttmejer A. The comparison of immunohistochemical proliferation and apoptosis markers in rectal carcinoma treated surgically or by preoperative radiochemotherapy. Pol J Pathol 2001; 52: 53-61.

27 Schelwies K, Sturm I, Grabowski P et al. Analysis of p53/BAX in primary colorectal carcinoma: low BAX protein expression is a negative prognostic factor in UICC stage III tumors. Int J Cancer 2002; 99: 589-96.

28 Nehls O, Okech T, Hsieh CJ et al. Low BAX protein expression correlates with disease recurrence in preoperatively irradiated rectal carcinoma. Int J Radiat Oncol Biol Phys 2005; 61: 85-91.

29 Scopa CD, Vagianos C, Kardamakis D et al. bcl-2/bax ratio as a predictive marker for therapeutic response to radiotherapy in patients with rectal cancer. Appl Immunohistochem Mol Morphol 2001; 9: 329-34.

30 Schott AF, Apel IJ, Nunez G et al. Bcl-XL protects cancer cells from p53-mediated apoptosis. Oncogene 1995; 11: 1389-94.

31 Fiebig AA, Zhu W, Hollerbach C et al. Bcl-XL is qualitatively different from and ten times more effective than $\mathrm{Bcl}-2$ when expressed in a breast cancer cell line. BMC Cancer 2006; 6: 213. 